\title{
Radiation in models with cosmological constant
}

\author{
Hedvika Kadlecová and Jiří Podolský \\ Institute of Theoretical Physics, Faculty of Mathematics and Physics, Charles University, \\ Prague, Czech Republic \\ email: Hedvika.Kadlecova@centrum.cz, podolsky@mbox.troja.mff.cuni.cz
}

\begin{abstract}
We analyze the asymptotic directional structure and properties of gravitational field of specific exact solutions belonging to the large family of black hole spacetimes of type $\mathrm{D}$ found by Plebański and Demiański. We discuss the structure in the case of de Sitter $(\Lambda>0)$ and antide Sitter $(\Lambda<0)$ conformal infinity. With the aim of further interpretation, we have found the relation between the structure of the sources (the mass $m$, the electric $e$ and magnetic $g$ charges, the NUT parameter $l$, the rotational parameter $a$, the acceleration $\alpha$ ) and the properties of radiation generated by them. In particular, we studied the amplitude of radiation and illustrated it in several figures.
\end{abstract}

Keywords. Plebański-Demiański family of solutions - asymptotic directional structure of radiation - de Sitter, anti-de Sitter

\section{Introduction}

The investigation of properties of gravitational radiation is an important problem of general relativity. In last few years, the theory was further developed for asymptotically de Sitter $(\Lambda>0)$ and anti-de Sitter $(\Lambda<0)$ spacetimes in Krtouš \& Podolský (2004). The work assumed, in analogy with the asymptotically flat situation, that the radiative field components are decaying as $\eta^{-1}$, where $\eta$ is an affine parameter along the null geodesics. We apply the new approach on particular exact model spacetimes of type D from the Plebański and Demiański family of solutions, concretely on the Kerr-Newmande Sitter metric and then on the general form of the metric which contains not only the mass, the charge and the rotation, but also the NUT parameter, non-zero acceleration and the cosmological constant, for details see Plebański \& Demiański (1976), Griffiths \& Podolský (2006). In both cases, we found the explicit dependence on the parameters of the metric in the final formulae of the directional structure of radiation.

\section{Results}

The general form of the metric has the form (Plebański \& Demiański 1976)

$$
\begin{aligned}
\mathbf{g}_{a b}= & \frac{1}{\Omega^{2}}\left[-\frac{Q}{\rho^{2}}\left(\mathrm{~d} \tilde{t}-\left(a \sin ^{2} \vartheta+4 l \sin ^{2} \frac{\vartheta}{2}\right) \mathrm{d} \tilde{\phi}\right)^{2}+\frac{\rho^{2}}{\hat{P}} \mathrm{~d} \vartheta^{2}+\frac{\rho^{2}}{Q} \mathrm{~d} r^{2}\right. \\
& \left.+\frac{\hat{P} \sin ^{2} \vartheta}{\rho^{2}}\left(\mathrm{~d} \tilde{t}-\left(r^{2}+(a+l)^{2}\right) \mathrm{d} \tilde{\phi}\right)^{2}\right],
\end{aligned}
$$

where $\Omega=1-\frac{\alpha}{\omega}(l+a \cos \vartheta), \rho^{2}=r^{2}+(l+a \cos \vartheta)^{2}, \hat{P}=1-a_{3} \cos \vartheta-a_{4} \cos ^{2} \vartheta$, $Q=\left(\omega^{2} k+e^{2}+g^{2}\right)-2 m r+\epsilon r^{2}-2 \alpha \omega^{-1} n r^{3}-\left(\alpha^{2} k+\Lambda / 3\right) r^{4}$. 

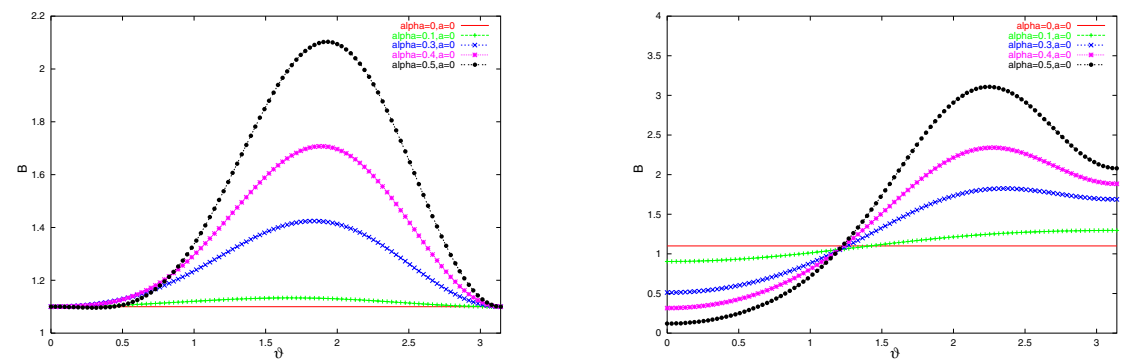

Figure 1. Left panel: the magnitude of radiation $B(\vartheta)$ on spacelike $\mathcal{I}$ for $\mathrm{C}-$ metric without charges, where $a=0$ and $\alpha$ varies. Right panel: the magnitude of radiation $B(\vartheta)$ on spacelike $\mathcal{I}$ for charged $\mathrm{C}-$ metric, where $a=0$ and $\alpha$ varies.

The directional pattern for $\Lambda>0$ is determined by two principal null directions (PNDs) oriented outwards with respect to the future spacelike conformal infinity $\mathcal{I}^{+}$, explicitly

$$
\left|\Psi_{4}^{\mathrm{i}}\right| \approx \frac{3}{2} \frac{1}{|\eta|} B(\vartheta) \mathcal{A}\left(\theta, \phi, \theta_{\mathrm{s}}\right),
$$

where $B(\vartheta)$ is the amplitude of radiation, which contain parameters of the metric. $\mathcal{A}\left(\theta, \phi, \theta_{\mathrm{s}}\right)$ is the directional structure, where $\theta$ and $\phi$ parametrize the direction along geodesic which approaches the conformal infinity, $\theta_{\mathrm{s}}$ is a parameter between two tetrads. In particular, for the $\mathrm{C}-$ metric the amplitude $B(\vartheta)$ has the form

$$
B(\vartheta)=\left(1+\frac{3}{\Lambda} \alpha^{2} \mathcal{P} \sin ^{2} \vartheta\right) \sqrt{m^{2}-4 m \alpha\left(e^{2}+g^{2}\right) \cos \vartheta+4 \alpha^{2}\left(e^{2}+g^{2}\right)^{2} \cos ^{2} \vartheta}
$$

where $\mathcal{P}=1-2 \alpha m \cos \vartheta+\alpha^{2}\left(e^{2}+g^{2}\right) \cos ^{2} \vartheta$. The dependence on parameters is illustrated in two graphs in Fig. 1. We verified results for C-metric obtained in Krtouš \& Podolský (2003) and in Podolský, Ortaggio \& Krtouš (2003). For Kerr-Newman-de Sitter, only the mass parameter occurs in the amplitude $B(\vartheta)=m$. We also investigated the structure near timelike $\mathcal{I}$, for details see Kadlecová (2006).

\section{Conclusions}

We investigated influence of the physical parameters of the sources, namely $\alpha, a, m, e$, $g$ and $\Lambda$ on the amplitude of the radiation $B(\vartheta)$ which appears at spacelike and timelike conformal infinity in the subcase $l=0$ of the general metric (2.1) and on its special subcases: the Kerr-Newman-de Sitter solution and the C-metric. Generally, we observe from Fig. 1 and other figures that the acceleration parameter $\alpha$ and the cosmological constant $\Lambda$ have dominant influence on the amplitude. The acceleration parameter $\alpha$ has stronger influence than the rotational parameter $a$. The charges $e$ and $g$ have a smaller influence than acceleration but their influence is quite stronger when the rotational parameter $a$ is small or vanishes. The asymptotic structure depends substantially on rotation when the acceleration is non-vanishing. We hope that these results will provide a deeper insight into the general theory of radiation in general relativity.

\section{References}

Plebański, J. F. \& Demiański, M. 1976, Ann. Phys. (NY), 98

Griffiths, J. B., Podolský, J. 2006, Int. J. Mod. Phys. D, 15, 335

Krtouš, P., Podolský, J. 2004, Class. Quantum Grav., 21, R233-273

Krtouš, P., Podolský, J. 2003, Phys. Rev. D, 68, 024005

Podolský, J., Ortaggio, M. \& Krtouš, P. 2003, Phys. Rev. D, 68, 124004

Kadlecová, H. 2006, Diploma Thesis, Charles Univesity, Prague 